Article

\title{
Application of Differential Transform to Multi-Term Fractional Differential Equations with Non-Commensurate Orders
}

\author{
Josef Rebenda \\ CEITEC BUT, Brno University of Technology, Purkynova 123, 61200 Brno, Czech Republic; \\ josef.rebenda@ceitec.vutbr.cz
}

Received: 30 September 2019; Accepted: 6 November 2019; Published: 9 November 2019

check for updates

\begin{abstract}
The differential transformation, an approach based on Taylor's theorem, is proposed as convenient for finding an exact or approximate solution to the initial value problem with multiple Caputo fractional derivatives of generally non-commensurate orders. The multi-term differential equation is first transformed into a multi-order system and then into a system of recurrence relations for coefficients of formal fractional power series. The order of the fractional power series is discussed in relation to orders of derivatives appearing in the original equation. Application of the algorithm to an initial value problem gives a reliable and expected outcome including the phenomenon of symmetry in choice of orders of the differential transformation of the multi-order system.
\end{abstract}

Keywords: fractional differential equation; non-commensurate orders; initial value problem; differential transform; fractional power series

\section{Introduction}

The main aim of this paper is to answer the open question mentioned in the quotation below: To find precise results about values of the constants in the formal asymptotic expansion of the solutions to initial value problems (IVPs) for multi-term fractional differential equations with generally non-commensurate orders. To explain the purpose of this paper-i.e., to give an answer to an open question-we need to place it in the context of analysis of multi-term fractional differential equations.

In Chapter 8 of the book [1] by Kai Diethelm, the author gave a thorough analysis to IVPs for multi-term fractional differential equations with Caputo derivatives, which in general may be non-commensurate.

The following quotation comes from Subchapter 6.5 of the above-mentioned book [1]:

"The theorems above have given us a large amount of information about the smoothness properties of the solutions of fractional differential equations, and in particular about the exact behaviour of the solution as $x \rightarrow 0$, most notably the formal asymptotic expansion. [...] An aspect of special significance, for example in view of the development of numerical methods, is the question for the precise values of the constants in this expansion, and most importantly the question whether certain coefficients vanish. A suitable generalisation of the Taylor expansion technique for ordinary differential equations described in [88, Chapter I.8] could be useful in this context. Precise results in this connection seem to be unknown at the moment though." 
An outline of the algorithm for IVP with two non-commensurate orders has been introduced in [2]. In addition, implementation of the algorithm to a concrete example suggested that there is certain symmetry in the possible choices of orders of the differential transformation used to turn the multi-order system into a system of recurrence relations. We observe the same phenomenon in the current paper.

The paper is organized as follows. Section 2 contains the problem statement and the main results including applications. In Section 3, we summarize the steps of the algorithm, discuss the results and outline possibilities of future work. The methods and tools necessary to obtain the results are recalled in Section 4.

\section{Results}

\subsection{Problem Statement}

As we want to use integer-order initial conditions which have clear practical meaning, we consider differential equations with Caputo fractional derivatives only. This way, we avoid issues with limit expressions present in fractional initial conditions that occur when the Riemann-Liouville definition is used. For the sake of clarity, we recall definition of the Caputo derivative.

Definition 1. The Caputo fractional derivative of order $\lambda$ (see, e.g., [3-5]) is defined by

$$
{ }_{t_{0}}^{C} D_{t}^{\lambda} f(t)=\frac{1}{\Gamma(n-\lambda)} \int_{t_{0}}^{t} \frac{f^{(n)}(s)}{(t-s)^{1+\lambda-n}} d s
$$

where $n-1 \leq \lambda<n, n \in \mathbb{N}, t>t_{0}$.

In this paper, we consider a class of multi-term fractional differential equations with non-commensurate orders in the form

$$
{ }_{0}^{C} D_{t}^{\lambda_{k}} y(t)=f\left(t, y(t){ }_{10}^{C} D_{t}^{\lambda_{1}} y(t){ }_{10}^{C} D_{t}^{\lambda_{2}} y(t), \ldots,{ }_{0}^{C} D_{t}^{\lambda_{k-1}} y(t)\right)
$$

with initial conditions

$$
y^{(i)}(0)=y_{0}^{(i)}, \quad i=0,1, \ldots,\left\lceil\lambda_{k}\right\rceil-1,
$$

where $\lambda_{k}>\lambda_{k-1}>\ldots>\lambda_{1}>0, \lambda_{j}-\lambda_{j-1} \leq 1$ for all $j=2,3, \ldots, k, 0<\lambda_{1} \leq 1,\lceil\cdot\rceil$ is the ceiling function, $f$ is an analytic function in some neighborhood of $\left(0, y_{0}^{(0)}, y_{0}^{(1)}, \ldots, y_{0}^{\left(\left\lceil\lambda_{k}\right\rceil-1\right)}\right)$ and ${ }_{0}^{C} D_{t}^{\beta}$ denotes the fractional derivative of order $\beta \in \mathbb{R}$ in Caputo sense. We assume that the orders of Equation (2) may in general be non-commensurate in the sense that $\frac{\lambda_{i}}{\lambda_{j}} \notin \mathbb{Q}$ for $i \neq j, i, j \in\{1, \ldots, k\}$.

\subsection{Algorithm Description}

Convenient approach to deal with equations of the type of Equation (2) is well described in the monograph by Diethelm [1]. Two ways to rewrite the multi-term fractional differential Equation (2) into a multi-order fractional differential system are presented in Chapter 8 and equivalence theorems are proved, where multi-order system means a system of single-order equations. Single-order equations of both rational and irrational order are analyzed in Chapter 6 of the same book. First, we combine information in both chapters to find a formal solution in the form of power series convergent in a neighborhood of the origin. Then, we apply the fractional differential transform (FDT) to find recurrence formula for coefficients of the power series. 


\subsubsection{Multi-Order System}

Theorem 1. Let $\lambda_{k}>\lambda_{k-1}>\ldots>\lambda_{1}>0, \lambda_{j}-\lambda_{j-1} \leq 1$ for all $j=2,3, \ldots, k, 0<\lambda_{1} \leq 1, \lambda_{i}$ and $\lambda_{j}$ generally be non-commensurate for $i \neq j$. Consider the IVP consisting of Equations (2) and (3), and assume that $f$ can be written in the form

$$
f\left(t, y(t){ }_{r}^{C} D_{t}^{\lambda_{1}} y(t), \ldots,{ }_{0}^{C} D_{t}^{\lambda_{k-1}} y(t)\right)=g\left(t, t^{\lambda_{1}}, \ldots, t^{\lambda_{k}}, y(t){ }_{0}^{C} D_{t}^{\lambda_{1}} y(t), \ldots,{ }_{0}^{C} D_{t}^{\lambda_{k-1}} y(t)\right)
$$

where $g$ is analytic in a neighborhood of $\left(0,0, \ldots, 0, y_{0}^{(0)}, y_{0}^{(1)}, \ldots, y_{0}^{\left(\left\lceil\lambda_{k}\right\rceil-1\right)}\right)$. Then, the solution $y$ can be written in the form

$$
y(t)=\sum_{j_{0}, j_{1}, \ldots, j_{k}=0}^{\infty} \bar{y}_{\left(j_{0}, j_{1}, \ldots, j_{k}\right)} t^{j_{0}+j_{1} \beta_{1}+\ldots+j_{k} \beta_{k}},
$$

where each coefficient $\bar{y}_{\left(j_{0}, j_{1}, \ldots, j_{k}\right)}$ is determined uniquely in terms of the coefficients corresponding to smaller exponents and the exponents $\beta_{j}, j=1, \ldots, k$ are defined as $\beta_{1}:=\lambda_{1}, \beta_{j}:=\lambda_{j}-\lambda_{j-1}, j=2,3, \ldots, k$.

Proof of Theorem 1. First, we need to rewrite Equation (2) in the form of multi-order system, i.e., a system of single-order equations of different orders, generally non-commensurate. We follow the approach described in [1] (p. 176).

We start by constructing a finite sequence of orders of the single-order equations, denote it $\left\{\lambda_{j}\right\}_{j=1}^{k}$. Without loss of generality, we can assume that all integers between 0 and $\lambda_{k}$ are members of the sequence too. Let us write $\beta_{1}:=\lambda_{1}, \beta_{j}:=\lambda_{j}-\lambda_{j-1}, j=2,3, \ldots, k$ and observe that for all $j, 0<\beta_{j} \leq 1$. Then, we may write $y_{1}:=y$ and $y_{j}:={ }_{0}^{C} D_{t}^{\beta_{j-1}} y_{j-1}, j=2,3, \ldots, k$. Applying Theorem 3 now, we conclude that the solution to the IVP defined by Equations (2) and (3) can be obtained from the solution to the system

$$
\begin{aligned}
{ }_{0}^{C} D_{t}^{\beta_{1}} y_{1}(t) & =y_{2}(t), \\
{ }_{0}^{C} D_{t}^{\beta_{2}} y_{2}(t) & =y_{3}(t), \\
\vdots & =\quad \vdots \\
{ }_{0}^{C} D_{t}^{\beta_{k-1}} y_{k-1}(t) & =y_{k}(t), \\
{ }_{0}^{C} D_{t}^{\beta_{k}} y_{k}(t) & =f\left(t, y_{1}(t), y_{2}(t), \ldots, y_{k}(t)\right)
\end{aligned}
$$

with the initial conditions

$$
y_{j}(0)= \begin{cases}y_{0}^{(0)} & \text { if } j=1 \\ y_{0}^{(i)} & \text { if } \lambda_{j-1}=i \in \mathbb{N} \\ 0 & \text { else }\end{cases}
$$

by setting $y:=y_{1}$. 
The next step is to rewrite the system of Equation (6) into an equivalent system of Volterra-type integral equations using Theorem 4

$$
\begin{aligned}
y_{1}(t) & =y_{0}^{(0)}+\frac{1}{\Gamma\left(\beta_{1}\right)} \int_{0}^{t}(t-s)^{\beta_{1}-1} y_{2}(s) \mathrm{d} s, \\
y_{2}(t) & =y_{0}^{\left(\lambda_{1}\right)}+\frac{1}{\Gamma\left(\beta_{2}\right)} \int_{0}^{t}(t-s)^{\beta_{2}-1} y_{3}(s) \mathrm{d} s, \\
\vdots & =\vdots \\
y_{k-1}(t) & =y_{0}^{\left(\lambda_{k-2}\right)}+\frac{1}{\Gamma\left(\beta_{k-1}\right)} \int_{0}^{t}(t-s)^{\beta_{k-1}-1} y_{k}(s) \mathrm{d} s, \\
y_{k}(t) & =y_{0}^{\left(\lambda_{k-1}\right)}+\frac{1}{\Gamma\left(\beta_{k}\right)} \int_{0}^{t}(t-s)^{\beta_{k}-1} f\left(s, y_{1}(s), y_{2}(s), \ldots, y_{k}(s)\right) \mathrm{d} s .
\end{aligned}
$$

Now, since we assume that the function $f$ can be written in the special form in Equation (4), we can apply Theorem 5 and Corollary 1 to each of the single-order equations in the system of Equation (6) with corresponding initial condition in Equation (7), one by one, to obtain

$$
y(t)=\sum_{j_{0}, j_{1}, \ldots, j_{k}=0}^{\infty} \bar{y}_{\left(j_{0}, j_{1}, \ldots, j_{k}\right)} t^{j_{0}+j_{1} \beta_{1}+\ldots+j_{k} \beta_{k}},
$$

where each coefficient $\bar{y}_{\left(j_{0}, j_{1}, \ldots, j_{k}\right)}$ is determined uniquely in terms of the coefficients corresponding to smaller exponents. The problem of how to determine the coefficients is the subject to our study in Section 2.2.2.

\subsubsection{Implementation of the Differential Transform}

Now, we turn our attention back to the IVP given by Equations (2) and (3). Recall that we have turned the problem into the equivalent IVP for a system of single-order fractional differential equations defined by Equations (6) and (7).

Applying the FDT tools developed in the Section 4.2, i.e., Theorem 6, the system of Equations (6) and (7) is transformed to the following system of recurrence relations

$$
\begin{aligned}
\frac{\Gamma\left(\alpha_{1} j_{1}+\beta_{1}+1\right)}{\Gamma\left(\alpha_{1} j_{1}+1\right)} Y_{1, \alpha_{1}}\left(j_{1}+\frac{\beta_{1}}{\alpha_{1}}\right) & =Y_{2, \alpha_{1}}\left(j_{1}\right), \\
\vdots & =\vdots \\
\frac{\Gamma\left(\alpha_{k} j_{k}+\beta_{k}+1\right)}{\Gamma\left(\alpha_{k} j_{k}+1\right)} Y_{k, \alpha_{k}}\left(j_{k}+\frac{\beta_{k}}{\alpha_{k}}\right) & =\mathcal{F}_{\alpha_{k}}\left(j_{k}, Y_{1, \alpha_{k}}\left(j_{k}\right), \ldots, Y_{k, \alpha_{k}}\left(j_{k}\right)\right),
\end{aligned}
$$

with transformed initial conditions

$$
Y_{j}(0)= \begin{cases}y_{0}^{(0)} & \text { if } j=1, \\ y_{0}^{(i)} / i ! & \text { if } \lambda_{j-1}=i \in \mathbb{N}, \\ 0 & \text { else, }\end{cases}
$$


where $\mathcal{F}_{\alpha_{k}}\left(j_{k}, Y_{1, \alpha_{k}}\left(j_{k}\right), \ldots, Y_{k, \alpha_{k}}\left(j_{k}\right)\right)$ is the FDT of $f\left(t, y_{1}(t), y_{2}(t), \ldots, y_{k}(t)\right)$ of order $\alpha_{k}$ and $0<\alpha_{1}, \ldots, \alpha_{k} \leq 1$ are suitable real constants representing the order of the fractional power series in Equation (27). If we had commensurate orders only, we could take all $\alpha_{1}, \ldots, \alpha_{k}$ equal to the least common multiple of denominators of all orders of derivatives which appear in the equation, as described in the paper [6]. However, in the case of non-commensurate orders, we have to use different approach for the choice of $\alpha_{1}, \ldots, \alpha_{k}$. Specifically, the choice $\alpha_{1}:=\beta_{1}, \ldots, \alpha_{k}:=\beta_{k}$ is one of the choices which lead to the solution of the given IVP in the form of Equation (5). The system of Equation (10) is then simplified to

$$
\begin{aligned}
\frac{\Gamma\left(\beta_{1} j_{1}+\beta_{1}+1\right)}{\Gamma\left(\beta_{1} j_{1}+1\right)} Y_{1, \beta_{1}}\left(j_{1}+1\right) & =Y_{2, \beta_{1}}\left(j_{1}\right), \\
\vdots & =\vdots \\
\frac{\Gamma\left(\beta_{k} j_{k}+\beta_{k}+1\right)}{\Gamma\left(\beta_{k} j_{k}+1\right)} Y_{k, \beta_{k}}\left(j_{k}+1\right) & =\mathcal{F}_{\beta_{k}}\left(j_{k}, Y_{1, \beta_{k}}\left(j_{k}\right), \ldots, Y_{k, \beta_{k}}\left(j_{k}\right)\right) .
\end{aligned}
$$

As there are (generally non-commensurate) different orders of the FDT in the system of Equation (12), we need to find relation between coefficients with different orders.

Theorem 2. Let a function $f$ have the form $f(t)={ }_{t_{0}}^{C} D_{t}^{\beta} g(t)$, where we allow $\beta=0$, and let $F_{\alpha_{1}}(k)$ and $F_{\alpha_{2}}(k)$ denote the FDT of $f$ at $t_{0}$ of orders $\alpha_{1}$ and $\alpha_{2}$, respectively. Then,

$$
F_{\alpha_{2}}(k)=F_{\alpha_{1}}\left(\frac{\alpha_{2}}{\alpha_{1}} k\right)
$$

Proof of Theorem 2. Application of Theorem 6 gives $F_{\alpha_{1}}(k)=\frac{\Gamma\left(\alpha_{1} k+\beta+1\right)}{\Gamma\left(\alpha_{1} k+1\right)} G_{\alpha_{1}}\left(k+\frac{\beta}{\alpha_{1}}\right)$ and $F_{\alpha_{2}}(k)=$ $\frac{\Gamma\left(\alpha_{2} k+\beta+1\right)}{\Gamma\left(\alpha_{2} k+1\right)} G_{\alpha_{2}}\left(k+\frac{\beta}{\alpha_{2}}\right)$. Calculating $G_{\alpha_{1}}$ and $G_{\alpha_{2}}$ from the Definition 2 we get

$$
\begin{aligned}
& G_{\alpha_{1}}\left(k+\frac{\beta}{\alpha_{1}}\right)=\frac{1}{\Gamma\left(\alpha_{1}\left(k+\frac{\beta}{\alpha_{1}}\right)+1\right)}\left[{ }_{t_{0}}^{C} D_{t}^{\alpha_{1}\left(k+\frac{\beta}{\alpha_{1}}\right)} g(t)\right]_{t=t_{0}}=\frac{1}{\Gamma\left(\alpha_{1} k+\beta+1\right)}\left[{ }_{t_{0}}^{C} D_{t}^{\alpha_{1} k+\beta} g(t)\right]_{t=t_{0}}{ }^{\prime} \\
& G_{\alpha_{2}}\left(k+\frac{\beta}{\alpha_{2}}\right)=\frac{1}{\Gamma\left(\alpha_{2}\left(k+\frac{\beta}{\alpha_{2}}\right)+1\right)}\left[{ }_{t_{0}}^{C} D_{t}^{\alpha_{2}\left(k+\frac{\beta}{\alpha_{2}}\right)} g(t)\right]_{t=t_{0}}=\frac{1}{\Gamma\left(\alpha_{2} k+\beta+1\right)}\left[{ }_{t_{0}}^{C} D_{t}^{\alpha_{2} k+\beta} g(t)\right]_{t=t_{0}} .
\end{aligned}
$$

Substituting $\frac{\alpha_{2}}{\alpha_{1}} k$ into $F_{\alpha_{1}}$ and combining all formulas brings us to the relation

$$
\begin{aligned}
F_{\alpha_{1}}\left(\frac{\alpha_{2}}{\alpha_{1}} k\right) & =\frac{\Gamma\left(\alpha_{1}\left(\frac{\alpha_{2}}{\alpha_{1}} k\right)+\beta+1\right)}{\Gamma\left(\alpha_{1}\left(\frac{\alpha_{2}}{\alpha_{1}} k\right)+1\right)} G_{\alpha_{1}}\left(\left(\frac{\alpha_{2}}{\alpha_{1}} k\right)+\frac{\beta}{\alpha_{1}}\right) \\
& =\frac{\Gamma\left(\alpha_{2} k+\beta+1\right)}{\Gamma\left(\alpha_{2} k+1\right)} \frac{1}{\Gamma\left(\alpha_{1}\left(\frac{\alpha_{2}}{\alpha_{1}} k\right)+\beta+1\right)}\left[{ }_{t_{0}}^{C} D_{t}^{\alpha_{1}\left(\frac{\alpha_{2}}{\alpha_{1}} k\right)+\beta} g(t)\right]_{t=t_{0}} \\
& =\frac{\Gamma\left(\alpha_{2} k+\beta+1\right)}{\Gamma\left(\alpha_{2} k+1\right)} \frac{1}{\Gamma\left(\alpha_{2} k+\beta+1\right)}\left[{ }_{t_{0}}^{C} D_{t}^{\alpha_{2} k+\beta} g(t)\right]_{t=t_{0}} \\
& =\frac{\Gamma\left(\alpha_{2} k+\beta+1\right)}{\Gamma\left(\alpha_{2} k+1\right)} G_{\alpha_{2}}\left(k+\frac{\beta}{\alpha_{2}}\right)=F_{\alpha_{2}}(k) .
\end{aligned}
$$


Theorem 2 allows us to solve the recurrence relations in the system of Equation (12) with respect to $j_{1}, \ldots, j_{k}$ to find the sequences of coefficients $\left\{Y_{1, \beta_{1}}\left(j_{1}\right)\right\}, \ldots,\left\{Y_{k, \beta_{k}}\left(j_{k}\right)\right\}$. Applying IFDT (Definition 3) yields

$$
y(t)=y_{1}(t)={ }^{C} \mathcal{D}_{\beta_{1}}^{-1}\left\{\left\{Y_{1, \beta_{1}}\left(j_{1}\right)\right\}_{j_{1}=0}^{\infty}\right\}[0]=\sum_{j_{1}=0}^{\infty} Y_{1, \beta_{1}}\left(j_{1}\right) t^{\beta_{1} j_{1}}=\sum_{j_{1}=0}^{\infty} Y_{1, \beta_{1}}\left(j_{1}\right) t^{\lambda_{1} j_{1}} .
$$

Although it seems that there are only powers of order $\lambda_{1}$ in the solution, it is not the case. Recall that, according to Definition 2 of FDT, indexes $j_{1}$ belong to a countable subset of $[0, \infty)$, not necessarily being integers. In fact, there will be integer multiples of all powers $\lambda_{1}, \ldots, \lambda_{k}$ and some integer powers of $t$ arising from the initial conditions in the solution, which is demonstrated in Section 2.2.3.

\subsubsection{Applications}

Example 1. Consider two-term fractional differential equation

$$
\Gamma\left(\frac{4}{3}\right){ }_{0}^{C} D_{t}^{\frac{1}{\sqrt{2}}} y(t)+\Gamma\left(\frac{1}{\sqrt{2}}+1\right){ }_{0}^{C} D_{t}^{\frac{1}{3}} y(t)=\Gamma\left(\frac{4}{3}+\frac{1}{\sqrt{2}}\right)\left(t^{\frac{1}{3}}+t^{\frac{1}{\sqrt{2}}}\right)
$$

with initial condition $y(0)=0$. If we rewrite Equation (15) into a two-order system, we get

$$
\begin{aligned}
{ }_{0}^{C} D_{t}^{\frac{1}{3}} y_{1}(t) & =y_{2}, \\
{ }_{0}^{C} D_{t}^{\frac{1}{\sqrt{2}}-\frac{1}{3}} y_{2}(t) & =\frac{1}{\Gamma\left(\frac{4}{3}\right)}\left(\Gamma\left(\frac{4}{3}+\frac{1}{\sqrt{2}}\right)\left(t^{\frac{1}{3}}+t^{\frac{1}{\sqrt{2}}}\right)-\Gamma\left(\frac{1}{\sqrt{2}}+1\right) y_{2}(t)\right),
\end{aligned}
$$

with initial conditions $y_{1}(0)=0, y_{2}(0)=0$. Following the algorithm, we choose $\alpha_{1}=\frac{1}{3}$ and $\alpha_{2}=\frac{1}{\sqrt{2}}-\frac{1}{3}$. Fractional differential transform of the system defined by Equations (16) and (17) is

$$
\begin{aligned}
\frac{\Gamma\left(\frac{1}{3} k+\frac{1}{3}+1\right)}{\Gamma\left(\frac{1}{3} k+1\right)} \Upsilon_{1, \alpha_{1}}(k+1) & =\Upsilon_{2, \alpha_{1}}(k) \\
\frac{\Gamma\left(\alpha_{2} k+\alpha_{2}+1\right)}{\Gamma\left(\alpha_{2} k+1\right)} \Upsilon_{2, \alpha_{2}}(k+1) & =\frac{1}{\Gamma\left(\frac{4}{3}\right)}\left(\Gamma\left(\frac{4}{3}+\frac{1}{\sqrt{2}}\right)\left(\delta\left(k-\frac{1}{3 \alpha_{2}}\right)+\delta\left(k-\frac{1}{\sqrt{2} \alpha_{2}}\right)\right)\right. \\
& \left.-\Gamma\left(\frac{1}{\sqrt{2}}+1\right) \Upsilon_{2, \alpha_{2}}(k)\right)
\end{aligned}
$$

with transformed initial conditions $Y_{1, \alpha_{1}}(0)=0, Y_{2, \alpha_{2}}(0)=0$. As Equation (19) does not depend on $Y_{1}$, we can solve it first completely and then get back to Equation (18). We observe that the first nonzero coefficient we get for $k=\frac{1}{3 \alpha_{2}}: Y_{2, \alpha_{2}}\left(\frac{1}{\sqrt{2} \alpha_{2}}\right)=\frac{\Gamma\left(\frac{4}{3}+\frac{1}{\sqrt{2}}\right)}{\Gamma\left(\frac{1}{\sqrt{2}}+1\right)}$. The next possibility of nonzero coefficient we observe for $k=\frac{1}{\sqrt{2} \alpha_{2}}$ :

$$
\Gamma\left(\frac{2}{\sqrt{2}}-\frac{1}{3}+1\right) \Upsilon_{2, \alpha_{2}}\left(\frac{2-\frac{\sqrt{2}}{3}}{1-\frac{\sqrt{2}}{3}}\right)=\frac{\Gamma\left(\frac{1}{\sqrt{2}}+1\right)}{\Gamma\left(\frac{4}{3}\right)}\left(\Gamma\left(\frac{4}{3}+\frac{1}{\sqrt{2}}\right)-\Gamma\left(\frac{1}{\sqrt{2}}+1\right) \Upsilon_{2, \alpha_{2}}\left(\frac{1}{\sqrt{2} \alpha_{2}}\right)\right)=0,
$$


which means that all coefficients $Y_{2, \alpha_{2}}(k)$ are zero except $Y_{2, \alpha_{2}}\left(\frac{1}{\sqrt{2} \alpha_{2}}\right)$. Finally we feed $Y_{2, \alpha_{2}}\left(\frac{1}{\sqrt{2} \alpha_{2}}\right)$ back into Equation (18) ( with $\left.k=\frac{3}{\sqrt{2}}\right)$ and obtain $Y_{1, \frac{1}{3}}\left(\frac{3}{\sqrt{2}}+1\right)=\frac{\Gamma\left(\frac{1}{\sqrt{2}}+1\right)}{\Gamma\left(\frac{4}{3}+\frac{1}{\sqrt{2}}\right)} Y_{2, \frac{1}{3}}\left(\frac{3}{\sqrt{2}}\right)=1$.

Hence, the IVP given by Equation (15) and the initial condition $y(0)=0$ has the unique solution

$$
y(t)=y_{1}(t)=t^{\frac{1}{3}\left(\frac{3}{\sqrt{2}}+1\right)}=t^{\frac{1}{3}+\frac{1}{\sqrt{2}}} .
$$

\section{Discussion}

In the paper, we propose an algorithm to obtain values of the constants in the formal asymptotic expansion of solution to IVP for multi-term fractional differential equation with generally non-commensurate orders. In particular, we proceed in the following sequence of steps:

1. Transform the multi-term equation into a multi-order differential system.

2. Describe an equivalent system of Volterra integral equations.

3. Find the general form of the asymptotic expansion of the solution.

4. Transform the multi-order differential system to a system of recurrence relations (formal application of FDT).

5. Choose convenient orders of FDT.

6. Describe the relation between different orders of FDT.

The algorithm provides an answer to the open question raised in the monograph by Diethelm [1]. An obvious subject to discuss is the choice of convenient orders of FDT (Step 5). Taking into account the symmetry observed in the choice of the orders in provided Example 1, we expect that there might be a different combination of orders used, with possibility to optimize the computational effort.

Further, convergence properties should be studied to ensure that a computer implementation of the algorithm is reliable and efficient. Once the convergence of the formal generalized power series is proved and an expression defining the radius of convergence is obtained, then we move in terminology from an asymptotic solution to an analytical approximate solution. This task can be a continuation of this article, with the proof of Theorem 6.32, p. 121-124, in [1] as a starting point.

Finally, as the orders are generally non-commensurate, i.e. irrational, software using symbolic computations might have an advantage against purely numerical software.

\section{Methods}

\subsection{Equivalence and Smoothness Theorems}

We recall a few results necessary for justification of correctness of the algorithm.

Theorem 3 (See [1], Theorem 8.9, p. 176). Subject to the conditions specified in Section 2.1, the multi-term Equation (2) with initial conditions in Equation (3) is equivalent to the system of Equation (6) with the initial conditions in Equation (7) in the following sense:

1. Whenever the function $y \in C^{\left\lceil\lambda_{k}\right\rceil}[0, T]$ is a solution of the IVP given by Equations (2) and (3), the vector-valued function $Y:=\left(y_{1}, \ldots, y_{k}\right)^{T}$ with

$$
y_{j}(t):= \begin{cases}y(t) & \text { if } j=1, \\ { }_{0}^{C} D_{t}^{\lambda_{j-1}} y(t) & \text { if } j=2, \ldots, k,\end{cases}
$$

is a solution of the IVP defined by Equations (6) and (7). 
2. Whenever the vector-valued function $Y:=\left(y_{1}, \ldots, y_{k}\right)^{T}$ is a solution of the IVP defined by Equations (6) and (7), the function $y:=y_{1}$ is a solution of the IVP given by Equations (2) and (3).

Theorem 4 (See [1], Lemma 6.2, p. 86). Let $0<n$ and $m=\lceil n\rceil$. Moreover, let $y_{0}^{(0)}, \ldots, y_{0}^{(m-1)} \in \mathbb{R}, K>0$ and $h^{*}>0$. Define $G:=\left\{(x, y): x \in\left[0, h^{*}\right],\left|y-\sum_{k=0}^{m-1} x^{k} y_{0}^{(k)} / k !\right| \leq K\right\}$, and let the function $f: G \rightarrow \mathbb{R}$ be continuous. The function $y \in C[0, h]$ for some $0<h<h^{*}$ is a solution of the initial value problem

$$
\begin{gathered}
{ }_{0}^{C} D_{x}^{n} y(x)=f(x, y(x)), \\
D^{k} y(0)=y_{0}^{(k)}, \quad k=0,1, \ldots, m-1
\end{gathered}
$$

if and only if it is a solution of the nonlinear Volterra integral equation of the second kind

$$
y(x)=\sum_{k=0}^{m-1} y_{0}^{(k)} \frac{x^{k}}{k !}+\frac{1}{\Gamma(n)} \int_{0}^{x}(x-t)^{n-1} f(t, y(t)) \mathrm{d} t .
$$

Theorem 5 (See [1], Theorem 6.35, p. 124). Let $n$ be a positive irrational number. Consider the initial value problem defined by Equations (22) and (23) and assume that $f$ can be written in the form $f(x, y)=\bar{f}\left(x, x^{n}, y\right)$ where $\bar{f}$ is analytic in a neighborhood of $\left(0,0, y_{0}^{(0)}\right)$. Then, there exists a uniquely determined analytic function $\bar{y}:(-r, r) \times\left(-r^{n}, r^{n}\right) \rightarrow \mathbb{R}$ with some $r>0$ such that $y(x)=\bar{y}\left(x, x^{n}\right)$ for $x \in[0, r)$.

Corollary 1 (See [1], Corollary 6.37, p. 125). Under the assumptions of Theorem 5, $y$ is of the form

$$
y(x)=\sum_{\mu, v=0}^{\infty} \bar{y}_{\mu v} x^{\mu+v n} .
$$

\subsection{Fractional Differential Transformation}

An introduction of a generalization of the differential transformation (DT) called the fractional differential transformation (FDT) is given in this subsection. For more details on DT and FDT, we recommend solid papers [7-12].

Definition 2. Fractional differential transformation of order $\alpha \in \mathbb{R}^{+}$of a real function $u(t)$ at a point $t_{0} \in \mathbb{R}$ in Caputo sense is ${ }^{C} \mathcal{D}_{\alpha}\{u(t)\}\left[t_{0}\right]=\left\{U_{\alpha}(k)\right\}_{k=0}^{\infty}, k \in I \subset \mathbb{R}_{0}^{+}$where $I$ is a countable subset of $[0, \infty)$, and $U_{\alpha}(k)$, the fractional differential transformation of order $\alpha$ of the $(\alpha k)$ th derivative of function $u(t)$ at $t_{0}$, is defined as

$$
U_{\alpha}(k)=\frac{1}{\Gamma(\alpha k+1)}\left[{ }_{t_{0}}^{C} D_{t}^{\alpha k} u(t)\right]_{t=t_{0}},
$$

provided that the original function $u(t)$ is analytic in some right neighborhood of $t_{0}$.

Definition 3. Inverse fractional differential transformation (IFDT) of $\left\{U_{\alpha}(k)\right\}_{k=0}^{\infty}$ is defined using a fractional power series as follows:

$$
u(t)={ }^{C} \mathcal{D}_{\alpha}^{-1}\left\{\left\{U_{\alpha}(k)\right\}_{k=0}^{\infty}\right\}\left[t_{0}\right]=\sum_{k=0}^{\infty} U_{\alpha}(k)\left(t-t_{0}\right)^{\alpha k}
$$

In applications, we use some basic FDT formulas listed in [6]: 
Theorem 6. Assume that $\left\{F_{\alpha}(k)\right\}_{k=0}^{\infty},\left\{G_{\alpha}(k)\right\}_{k=0}^{\infty}$ and $\left\{H_{\alpha}(k)\right\}_{k=0}^{\infty}$ are differential transformations of order $\alpha$ at $t_{0}$ of functions $f(t), g(t)$ and $h(t)$, respectively. Further assume that $r>0, \beta>0$.

$$
\begin{aligned}
& \text { If } f(t)=\left(t-t_{0}\right)^{r} \text {, then } F_{\alpha}(k)=\delta\left(k-\frac{r}{\alpha}\right) \text {, where } \delta(x-y)=\delta_{x y} \text { is the Kronecker delta. } \\
& \begin{array}{l}
\text { If } f(t)=g(t) h(t) \text {, then } F_{\alpha}(k)=\sum_{l=0}^{k} G_{\alpha}(l) H_{\alpha}(k-l) . \\
\text { If } f(t)=\frac{g(t)}{\left(t-t_{0}\right)^{r}} \text {, then } F_{\alpha}(k)=G_{\alpha}\left(k+\frac{r}{\alpha}\right) \text {, provided } G_{\alpha}(l)=0 \text { for } l<\frac{r}{\alpha} . \\
\text { If } f(t)={ }_{t_{0}}^{C} D_{t}^{\beta} g(t), \text { then } F_{\alpha}(k)=\frac{\Gamma(\alpha k+\beta+1)}{\Gamma(\alpha k+1)} G_{\alpha}\left(k+\frac{\beta}{\alpha}\right) .
\end{array}
\end{aligned}
$$

Acknowledgments: The author was supported by the Czech Science Foundation under the project 19-23815S. The Article Processing Charge was funded by Open Access Fund of Brno University of Technology. This support is gratefully acknowledged.

Conflicts of Interest: The author declares no conflict of interest. The funders had no role in the design of the study; in the collection, analyses, or interpretation of data; in the writing of the manuscript, or in the decision to publish the results.

\section{Abbreviations}

The following abbreviations are used in this manuscript:

IVP Initial Value Problem

DT Differential Transformation

FDT Fractional Differential Transformation

IFDT Inverse Fractional Differential Transformation

\section{References}

1. Diethelm, K. The Analysis of Fractional Differential Equations: An Application-Oriented Exposition Using Differential Operators of Caputo Type; Springer: Berlin, Germany, 2010.

2. Rebenda, J.; Šmarda, Z. Application of differential transform to two-term fractional differential equations with noncommensurate orders. In Proceedings of the International Conference of Numerical Analysis and Applied Mathematics (ICNAAM 2018), Rhodes, Greece, 13-18 September 2018; Simos, T.E., Ed.; AIP Publishing: Melville, NY, USA, 2019; Volume 2116, p. 310008. [CrossRef]

3. Podlubny, I. Fractional Differential Equations; Academic Press: New York, NY, USA, 1999.

4. Kilbas, A.A.; Srivastava, H.M.; Trujillo, J.J. Theory and Applications of Fractional Differential Equations; Elsevier: Amsterdam, The Netherlands, 2006.

5. Kaczorek, T.; Rogowski, K. Fractional Linear Systems and Electrical Circuits. In Studies in Systems, Decision and Control; Springer International Publishing: Cham, Switzerland, 2015; Volume 13.

6. Rebenda, J.; Šmarda, Z. A numerical approach for solving of fractional Emden-Fowler type equations. In Proceedings of the International Conference of Numerical Analysis and Applied Mathematics (ICNAAM 2017), Thessaloniki, Greece, 25-30 September 2017; Simos, T.E., Ed.; AIP Publishing: Melville, NY, USA, 2018; Volume 1978, p. 140006.

7. Šamajová, H.; Li, T. Oscillators near Hopf bifurcation. Commun. Sci. Lett. Univ. Žilina 2015, 17, 83-87.

8. Rebenda, J.; Šmarda, Z. A differential transformation approach for solving functional differential equations with multiple delays. Commun. Nonlinear Sci. Numer. Simul. 2017, 48, 246-257. [CrossRef]

9. Rebenda, J.; Šmarda, Z. Numerical algorithm for nonlinear delayed differential systems of $n$th order. Adv. Differ. Equ. 2019, 2019, 26. [CrossRef] 
10. Šamajová, H. Semi-Analytical Approach to Initial Problems for Systems of Nonlinear Partial Differential Equations with Constant Delay. In Proceedings of the EQUADIFF 2017 Conference, Bratislava, Slovakia, 24-28 July 2017; Mikula, K., Sevcovic, D., Urban, J., Eds.; Spektrum STU Publishing: Bratislava, Slovakia, 2017; pp. 163-172.

11. Yang, X.J.; Tenreiro Machado, J.A.; Srivastava, H.M. A new numerical technique for solving the local fractional diffusion equation: Two-dimensional extended differential transform approach. Appl. Math. Comput. 2016, 274, 143-151. [CrossRef]

12. Rebenda, J.; Šmarda, Z. Numerical Solution of Fractional Control Problems via Fractional Differential Transformation. In Proceedings of the 2017 European Conference on Electrical Engineering and Computer Science (EECS), Bern, Switzerland, 17-19 November 2017; pp. 107-111. [CrossRef]

(C) 2019 by the author. Licensee MDPI, Basel, Switzerland. This article is an open access article distributed under the terms and conditions of the Creative Commons Attribution (CC BY) license (http:/ / creativecommons.org/licenses/by/4.0/). 